\title{
Acceleration of Polarized Protons in the AGS
}

N. Tsoupas, L. Ahrens, M. Bai, K. Brown, E. Courant, J.W. Glenn, H. Huang, A. Luccio, W.W. MacKay, T. Roser, V. Schoefer, K. Zeno

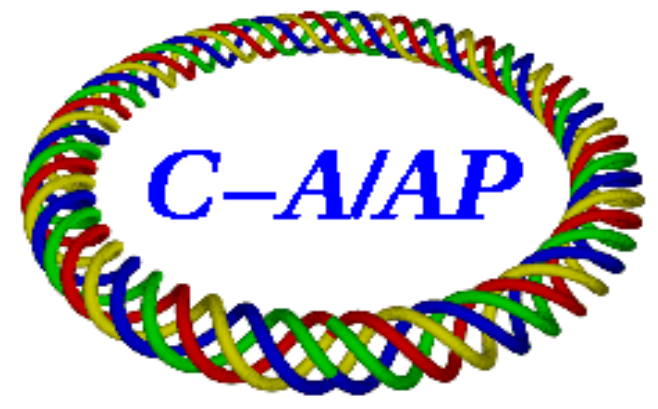

\section{Collider-Accelerator Department Brookhaven National Laboratory Upton, NY 11973}

Notice: This document has been authorized by employees of Brookhaven Science Associates, LLC under Contract No. DE-AC02-98CH10886 with the U.S. Department of Energy. The United States Government retains a nonexclusive, paid-up, irrevocable, world-wide license to publish or reproduce the published form of this document, or allow others to do so, for United States Government purposes. 


\title{
Acceleration of polarized protons in the AGS
}

\author{
N. Tsoupas, L. Ahrens, M. Bai, K. Brown, E. Courant, J. W. Glenn, H. Huang, \\ A. Luccio, W. W. MacKay, T. Roser, V. Schoefer, K. Zeno, BNL
}

\begin{abstract}
The high energy $\left(s^{1 / 2}=500 \mathrm{GeV}\right)$ polarized proton beam experiments performed in RHIC, require high polarization of the proton beam. With the AGS used as the pre-injector to RHIC, one of the main tasks is to preserve the polarization of the proton beam, during the beam acceleration in the AGS. The polarization preservation is accomplished by the two partial helical magnets $[1,2,3,4,5,6,7]$ which have been installed in AGS, and help overcome the imperfection and the intrinsic spin resonances which occur during the acceleration of protons. This elimination of the intrinsic resonances is accomplished by placing the vertical tune $\mathrm{Q}_{\mathrm{y}}$ at a value close to 8.98, within the spin-tune stop-band created by the snake. At this near integer tune the perturbations caused by the partial helical magnets is large resulting in large beta and dispersion waves. To mitigate the adverse effect of the partial helices on the optics of the AGS, we have introduced compensation quads[2] in the AGS. In this paper we present the beam optics of the AGS which ameliorates this effect of the partial helices.
\end{abstract}

\section{Introduction}

The high energy $\left(s^{1 / 2}=500 \mathrm{GeV}\right)$ polarization experiments performed in RHIC require high beam polarization. One of the requirements of high beam polarization, is to inject into RHIC from the AGS, which acts as a pre-injector to RHIC, a highly polarized beam. The preservation of the polarization, of a polarized proton beam, during the acceleration of the beam in the AGS is not a trivial task since the polarized beam encounters many "spin- resonances" which may depolarize the beam. In order to overcome these spin resonances which cause depolarization of the polarized beam, it was suggested [1] of inserting partial helical magnets in the AGS. These partial helical magnets, act both, as strong artificial resonances which minimize the polarization loss at the imperfection resonances, and also eliminate the intrinsic spin resonances. This idea of using multiple helices, was applied to the AGS synchrotron [2,3] by inserting into the AGS ring two partial helices, and it proved to be successful. Although the high field partial helices are beneficial for the preservation of the beam polarization during the beam acceleration, the high fields of the helices generate local beam bumps, distort the betatron $\left(\beta_{\mathrm{x}, \mathrm{y}}\right)$ and dispersion $\left(\eta_{\mathrm{x}, \mathrm{y}}\right)$ functions, and also introduce linear beam coupling. These effects, introduced by the partial helices on the beam optics, can be mitigated by introducing compensation quadrupoles at the vicinity of the partial helices [3] and by generating local beam bumps at the location of the partial helices. During the beam acceleration both partial helices maintain a constant field, therefore the adverse effect of the helices on the beam optics of the AGS is significant at low beam energies and diminishes as the inverse of the beam momentum, becoming almost insignificant at the extraction energies of p 25.5 GeV/c. Similarly the strength of the compensation quadrupoles, which help 
minimize the effect of the helices, is reduced by the inverse of the square of the momentum $\left(1 / \mathrm{p}^{2}\right)$. In this paper we present the beam optics of the AGS as a function of the beam momentum, and we also discuss the various beam constraints that should be satisfied during the beam acceleration.

\section{Layout of the two Helices and of the compensation Quads in AGS.}

In this section we discuss the Layout of the various devices which have been introduced in the AGS to preserve the beam polarization during the acceleration cycle by satisfying the constraints imposed on the beam to accomplish both polarization preservation and reasonable beam optics with minimal beam losses.

Table 1. The location of the Cold, and Warm helices, and the location of the compensation quads in AGS is shown in $2^{\text {nd }}$ column. The type of magnet is shown in shown in the $3^{\text {rd }}$ column.

\begin{tabular}{|c|c|c|c|c|c|}
\hline $\begin{array}{c}\text { Element } \\
\text { Number }\end{array}$ & $\begin{array}{c}\text { Element } \\
\text { Name }\end{array}$ & $\begin{array}{c}\text { Straight section } \\
\text { Location }\end{array}$ & Description & Power Supply & $\begin{array}{c}\text { Optical } \\
\text { Properties }\end{array}$ \\
\hline 1 & CQ_A17 & SS-A17 & Tune Quad QH_A17 & $\begin{array}{c}\text { Floating PS } \\
\text { across Quad }\end{array}$ & Defocusing \\
\hline 2 & CQ_A19 & SS-A19 & Thin Quad & Independent PS & Defocusing \\
\hline 3 & Cold Helix & SS-A20 & $\begin{array}{c}\text { Super Conducting } \\
\text { Helical Magnet }\end{array}$ & Power Supply & $\begin{array}{c}\text { Focusing in } \\
\text { both planes }\end{array}$ \\
\hline 4 & CQ_B1 & SS-B1 & Thin Quad & Independent PS & Defocusing \\
\hline 5 & CQ_B3 & SS-B3 & Polarization Quad & $\begin{array}{c}\text { Floating PS } \\
\text { across Quad }\end{array}$ & Defocusing \\
\hline 6 & CQ_E17 & SS-E17 & Tune Quad QH_E17 & $\begin{array}{c}\text { Floating PS } \\
\text { across Quad }\end{array}$ & Defocusing \\
\hline 7 & CQ_E19 & SS-E19 & $\begin{array}{c}\text { Thin Quad in series } \\
\text { with CQ-F1 }\end{array}$ & Independent PS & Defocusing \\
\hline 8 & Warm Helix & SS-E20 & $\begin{array}{c}\text { Warm Helical } \\
\text { Magnet }\end{array}$ & Power Supply & $\begin{array}{c}\text { Focusing in } \\
\text { both planes }\end{array}$ \\
\hline 9 & CQ_F1 & SS-F1 & $\begin{array}{c}\text { Thin Quad in series } \\
\text { with CQ-E19 }\end{array}$ & Independent PS & Focusing \\
\hline 10 & CQ_F3 & SS-F3 & Polarization Quad & No PS & None \\
\hline
\end{tabular}

Table 1 provides information on the location of the Cold and Warm helices (Element numbers 3 and 8) and the location of the compensation quadrupoles. The first letter "C" of the element name in the $2^{\text {nd }}$ column indicates “Compensation Quad". Among the compensation quads, those corresponding to the element numbers 2,4,7, and 9, (see Table 1) are independent "thin" quadrupoles [8], and those appearing in element numbers 1, 5 and 6 are the regular Tune Quads in AGS (el.\#. 1,6) and Polarization Quad (el. \# 5), each, with a "floating" power supply across each quadrupole for independent control of these particular quads.

Note that the present optics did not require independent control of the polarization quad (el. \# 10). Also the string of the polarization quads in AGS which are located in the 
Straight Sections 03 (SS-03) of every other super-period of AGS, are connected in series with the Vertical tune quads which are located in the straight section 03 (SS-03) of every super-period in AGS.

\section{Beam Constraints of AGS during the acceleration of protons.}

Two main beam constraints should be satisfied during the acceleration of polarized protons. The first constraint requires that the maximum beam size, fits into the available aperture of the accelerator. The second constrain, which is relevant to the preservation of the polarizatio, requires that the vertical tune maintains a value of 8.97 or higher during the acceleration. The first constrain is satisfied by maintaining the maximum values of the beta functions $\beta_{\mathrm{x}, \mathrm{y}}$ and $\eta_{\mathrm{x}, \mathrm{y}}$ below a specified limit.

For example at injection we apply the following constraints on the $\beta_{\mathrm{x}, \mathrm{y}}$ and $\eta_{\mathrm{x}, \mathrm{y}}$ functions: $\beta_{\mathrm{x}, \mathrm{y}}<50[\mathrm{~m}]$, and $\left\{-4[\mathrm{~m}]<\eta_{\mathrm{x}}<-0.5[\mathrm{~m}]\right\}$.

Assuming an rms value for the normalized beam emittance, of $\varepsilon_{\mathrm{x}, \mathrm{y}} \sim 1.7 \pi[\mathrm{mm} . \mathrm{mrad}]$ and an rms value for the momentum spread of $\sigma_{\mathrm{p}} \sim 7 \times 10^{-4}$, then the maximum rms values of the beam size $\left(\sigma_{\max }\right)_{\mathrm{x}}$, and $\left(\sigma_{\max }\right)_{\mathrm{y}}$, at injection $(\mathrm{G} \gamma=4.5, \beta \gamma=2.3)$ is:

$\left(\sigma_{\max }\right)_{\mathrm{x}} \leq\left\{\left(\varepsilon_{\mathrm{x}, \mathrm{y}} \cdot \beta_{\mathrm{x}, \mathrm{y}}\right) /(\beta \gamma)+\left(\eta_{\mathrm{x}} \cdot \sigma_{\mathrm{p}}\right)^{2}\right\}^{1 / 2}=0.67[\mathrm{~cm}]$ and $\left(\sigma_{\max }\right)_{\mathrm{y}} \leq 0.61[\mathrm{~cm}]$

In the expressions above, the quantities $\left(\sigma_{\max }\right)_{x}$, and $\left(\sigma_{\max }\right)_{y}$ are the rms values of the horizontal and vertical beam sizes, and the symbols, $\gamma$ and $\beta$, are the well known relativistic quantities.

It is understood that as the beam is accelerated, the geometrical emittance becomes smaller $\left\{\left.\varepsilon_{\mathrm{x}, \mathrm{y}}\right|_{\text {geom. }}=\left.\varepsilon_{\mathrm{x}, \mathrm{y}}\right|_{\text {Norm }} /(\beta \gamma)\right\}$ therefore we are allowed to relax the constraint on the maximum value of the $\beta_{\mathrm{x}, \mathrm{y}}$ functions.

\section{Beam Optics of the AGS during the acceleration of protons.}

The beam optics of the polarized beam in the AGS synchrotron during the acceleration is calculated using a MAD model of the AGS which is used by the "MAD" code (mad8b) [12] to generate the settings of the compensation quads and the settings of the tune quads for the beam to satisfy the constraints mentioned earlier.

Below we describe the details of the procedure we follow to set up the AGS synchrotron for the acceleration of the proton beam, and also provide Tables with the required settings of the compensation quads and tune quads to satisfy the beam constraints for the acceleration of protons.

The optical properties of both, the Warm and Cold helices are provided in the MAD model of the AGS synchrotron, in the form of 6x6 R-matrices. These R-matrices have been calculated by raytracing charged particles in the 3D magnetic field maps of the helices. These three dimensional magnetic field maps of the helices have been calculated using the "TOSCA" module (magnetostatic) of the OPERA [11] computer code for electromagnetics. 
During the beam acceleration the field strength of both, the Warm and Cold helices remains constant, therefore as the momentum of the protons increases, the trajectory of the protons inside the helical magnet will be different. Since the field of the helical magnets is not linear, it is required that the R-matrices for the Warm and the Cold helices are calculated for each beam momentum during acceleration. Since the strength of each helix remains the same during the acceleration, the effect of the helices on the beam optics, is reduced inversely proportional to the beam rigidity. This is not the case however with the effect of the helices on the spin rotation which remains almost constant as the beam momentum increases. This is a consequence of the Thomas-BMT equation $[9,10]$ which describes the motion of the magnetic moment of a particle in an external magnetic field.

In the rest of the section we describe the procedure we follow to obtain the settings of the various devices in the AGS which help satisfy the beam constrains, we mention earlier, during the beam acceleration.

Procedure:

1. A MAD model of the AGS synchrotron, which includes the cold and warm helices, and also includes the compensation quads, is read by the mad8b computer code. As we mentioned earlier, both the cold and warm helices appear in the MAD model in the form of $6 \times 6$ linear matrices.

2. Starting at injection energy, the mad8b code closes the orbit, and imposes the beam constraints of aperture and vertical tunes.

3. The momentum of the proton beam is subsequently increased by steps corresponding to steps of $\Delta \gamma=0.1$ ( $\gamma$ is the relativistic Lorentz factor), the Rmatrices of both helices are updated to those corresponding to this particular momentum, and the mad8b code repeats step 2 above.

4. The step 3 is repeated until the beam momentum reaches the extraction momentum which corresponds to a value of $\gamma=25.3(\mathrm{G} \gamma=45.4)$.

During the acceleration, the beam rigidity increases therefore the optical effect of the helices on the beam optics is reduced. This in turn reduces the strength of the compensation quadrupoles which are exclusively used to correct for the effect of the helices on the optics of the beam. When the beam momentum reaches the value which corresponds to the value of $\gamma \approx 6.0$, the strength of all compensation quads is zero and the R-matrices of the helices almost resemble the matrices of drift space.

Fig. 1 shows the beta $\left(\beta_{x, y}\right)$ and, dispersion $\left(\eta_{x, y}\right)$ functions along the AGS. The values of the beta functions $\left(\beta_{x, y}\right)$ are constraint to be below the value of $50 \mathrm{~m}$ and the dispersion $\left(\eta_{\mathrm{x}, \mathrm{y}}\right)$ functions are constrained in the range $\left\{-4[\mathrm{~m}]<\eta_{\mathrm{x}}<-0.5[\mathrm{~m}]\right\}$ throughout the AGS ring. The blue dashed line in figure 1 is the vertical dispersion which is generated by the assumed linear coupling of the warm and cold helices, and this coupling is introduced through the R-matrices. At higher energies, the beta and dispersion functions do satisfy the required constraints and at $\gamma \approx 6.0$, they converge to the values of the regular AGS because the strength of the helices is a small perturbation to the beam 


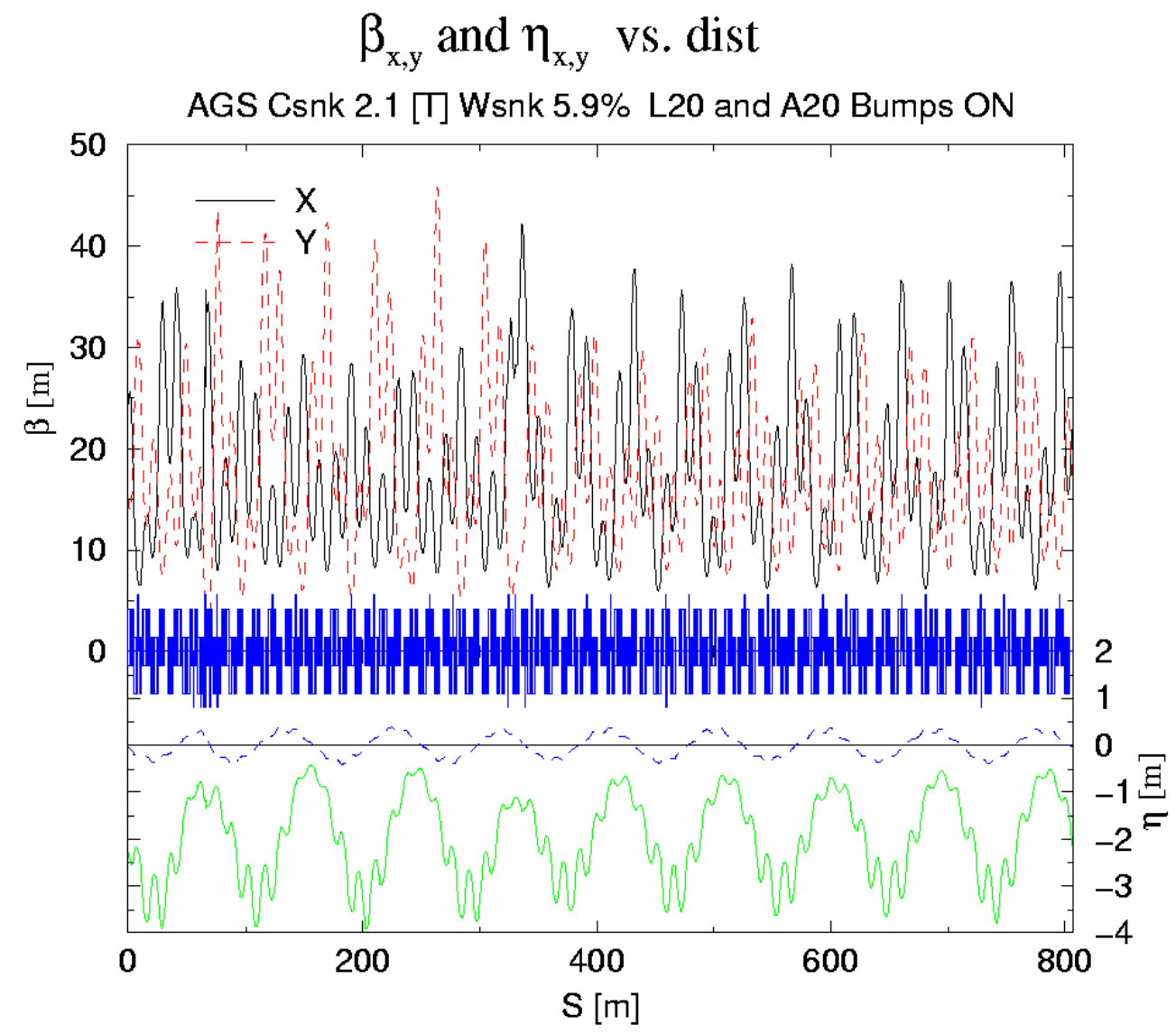

Figure 1. The beta $\left(\beta_{x, y}\right)$ functions, and the dispersion functions $\left(\eta_{x, y}\right)$ at injection energy $(G \gamma=4.5)$ plotted along the AGS. Note that the blue dashed line is the vertical dispersion function generated by the linear coupling of the warm and cold helices. This linear coupling is introduced through the R-matrices which represent the helices in the MAD lattice of the AGS.

During injection we excite two beam bumps, the "L20 Bump", and the "Cold helix A20 Bump". The "L20 Bump" consist of the "L20 position Bump" and the "L20 Angle Bump", and it is used in conjunction with the "L20 Septum" and the "A5_kicker" during beam injection. The "L20 Bump" is generated by a set of backleg windings placed on specific main magnets of the AGS. A schematic diagram of these main magnets which have the backleg windings, is shown in figure 2. The "Cold helix A20 Bump", bumps the beam at the location of the Cold helix, to counterbalance the displacement of the beam which is caused by the Cold helix. Like the "A20 Bump", this "Cold helix A20 
Bump”, is generated by a set of backleg windings placed on specific main magnets of the AGS. A schematic diagram of these main magnets is shown in figure 2.

\section{Arrangement of the Backleg Windings for the L20 (Position, Angle) and A20 (Csnk) Bumps}
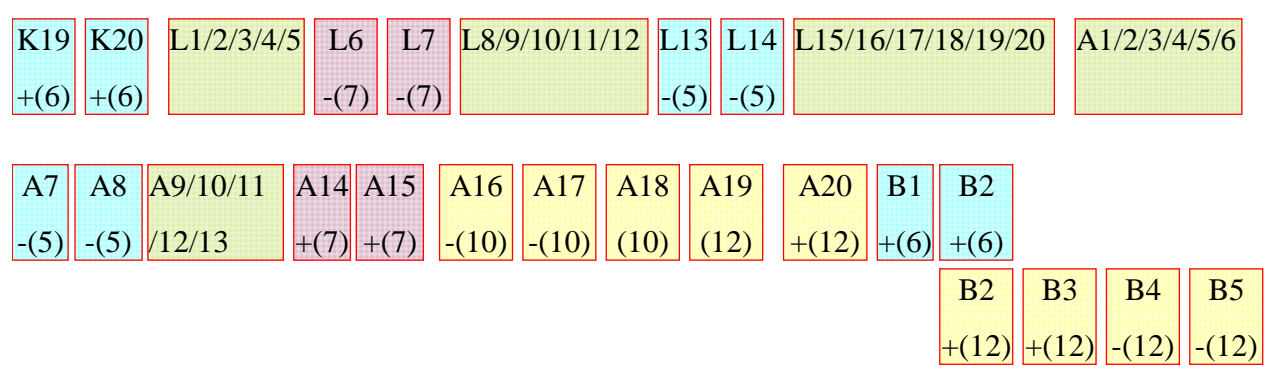

\begin{tabular}{|c|}
\hline L20 Position \\
\hline L20 Angle \\
\hline A20 Cold Snake \\
\hline
\end{tabular}

Figure 2. A schematic diagram showing the main magnets which carry the backleg windings to generate the "L20 Bump", and the "Cold helix A20 Bump". The numbers in parenthesis indicate the number of turns of each winding. The sign in front of the magnet indicates the direction of the winding.

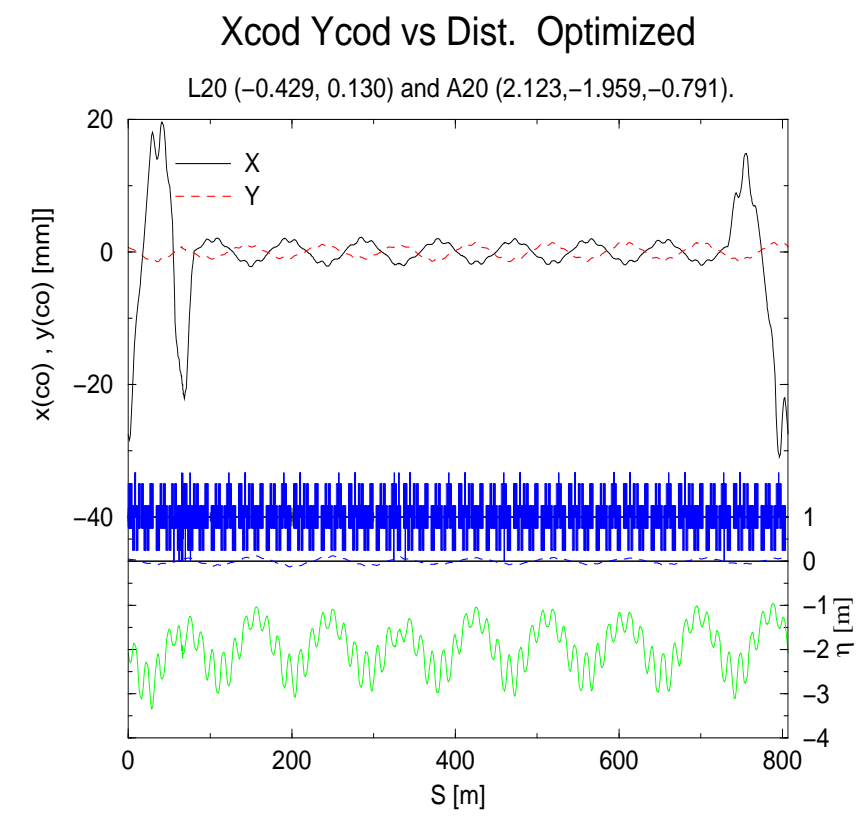

Time: Tue Sep 30 15:56:15 2008 Last file modify time: Tue Sep 30 14:16:06 2008

Figure 3. The horizontal $X_{\text {cod }}$, and vertical $Y_{\text {cod }}$ closed orbit beam displacements at beam injection in AGS. The "L20 Bump", and the "Cold helix A20 Bump" are both excited. 
Figure 3 shows the Horizontal closed orbit displacement $\left(\mathrm{X}_{\mathrm{cod}}\right)$, and the vertical closed orbit displacement ( $\left.\mathrm{Y}_{\mathrm{cod}}\right)$, at beam injection in AGS. The $\mathrm{X}_{\mathrm{cod}}$, is generated by the excitation of the "L20 Bump", and the "Cold helix A20 Bump" (see text). The linear coupling introduced by the helices generates the nonzero value of the $\mathrm{Y}_{\text {cod }}$.

The "L20 Bump” is reduced to zero just after injection, and the "Cold helix A20 Bump" remains excited, but with its strength diminishing gradually to zero when the energy of the beam reaches a value which corresponds to $\gamma \approx 6.0$.

Figures 4 and 5 show the maximum value of the $\beta$ function and the tune $Q$ for the horizontal and vertical planes respectively as a function of $G \gamma$. The spikes appearing in the tune, $\mathrm{Q}_{\mathrm{x}}$, of figure 4 were introduced by the MAD fitting program, and can be corrected, by slightly varying the strength of the Horizontal or vertical Quadrupoles. In Figures 4 and 5, the red line corresponds to the AGS optics where we constrain the $\mathrm{Q}_{\mathrm{y}}$ tunes to have the value of 8.985 for $G \gamma>7.5$, and the black line corresponds to the AGS optics where we constrain the $\mathrm{Q}_{\mathrm{y}}$ tunes to have the value of 8.980 for $\mathrm{G} \gamma>7.5$. Although the linear coupling requires running the mad8b code in the "coupled mode", it appears that the coupling is very weak, and for all practical purposes the results of running the code in the "coupled mode" are almost identical to those when we run the code in the "uncoupled mode". We have chosen the uncoupled mode because it alows us to use the optimization of the mad8b code.

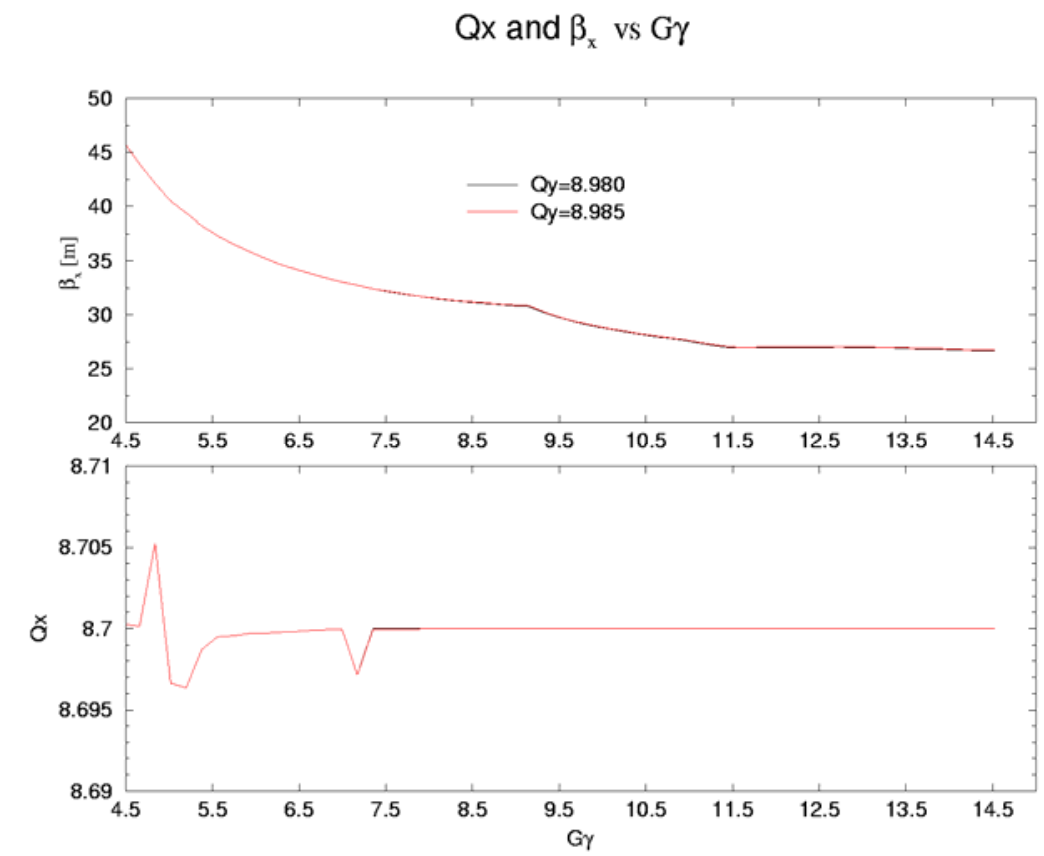

Figure 4. The beta function $\left(\beta_{\mathrm{x}}\right)$ top plot, and the horizontal tune $\mathrm{Q}_{\mathrm{x}}$ as a function of $\mathrm{G} \gamma$. 
The spikes appearing in the tune, $\mathrm{Q}_{\mathrm{x}}$, were introduced by the MAD fitting program, and can be corrected, by only slightly varyin the strength of the Horizontal or vertical Quadrupoles.

\section{Qy and $\beta_{y}$ vs $G \gamma$}
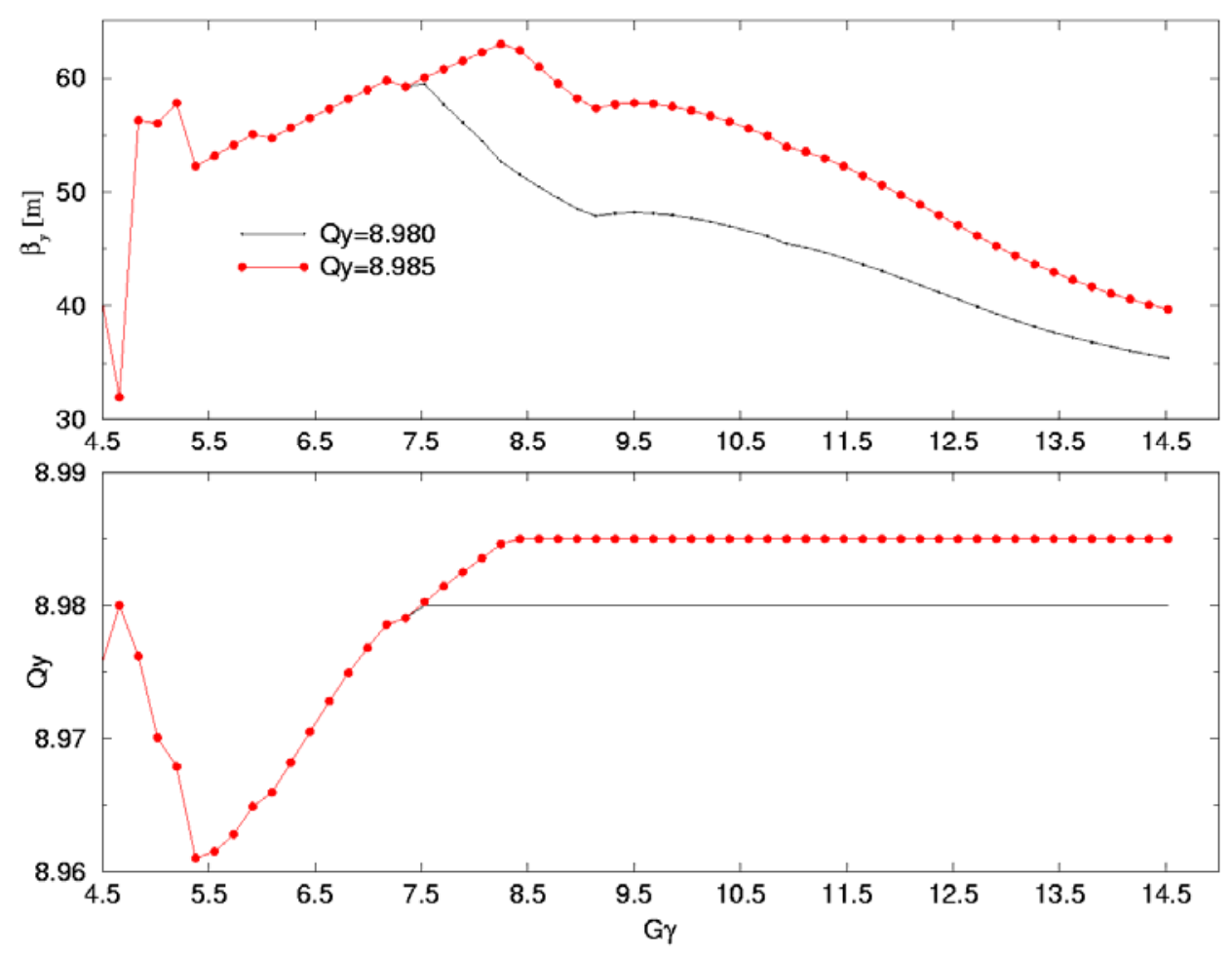

Figure 5. The beta function ( $\beta_{\mathrm{y}}$ ) top plot, and the vertical tune $\mathrm{Q}_{\mathrm{y}}$ as a function of $\mathrm{G} \gamma$. The red line corresponds to the AGS optics where we constrain the $\mathrm{Q}_{\mathrm{y}}$ tunes to have the value of 8.985 for $G \gamma>7.5$, and the black line corresponds to the AGS optics where we constrain the $\mathrm{Q}_{\mathrm{y}}$ tunes to have the value of 8.980 for $\mathrm{G} \gamma>7.5$.

\section{Settings of the compensation quads and of the tune quads during the acceleration of the polarized protons.}

In this section we will present the settings of the compensation quads as they are calculated from the mad8b code during the acceleration. As we mentioned earlier the constraints we impose on the beam are being used in the mad8b code.

Figure 6 shows the plots the current of the horizontal (top) and vertical (bottom) tune quadrupoles as a function of $\mathrm{G} \gamma$. 
I vs $\mathrm{G} \gamma$

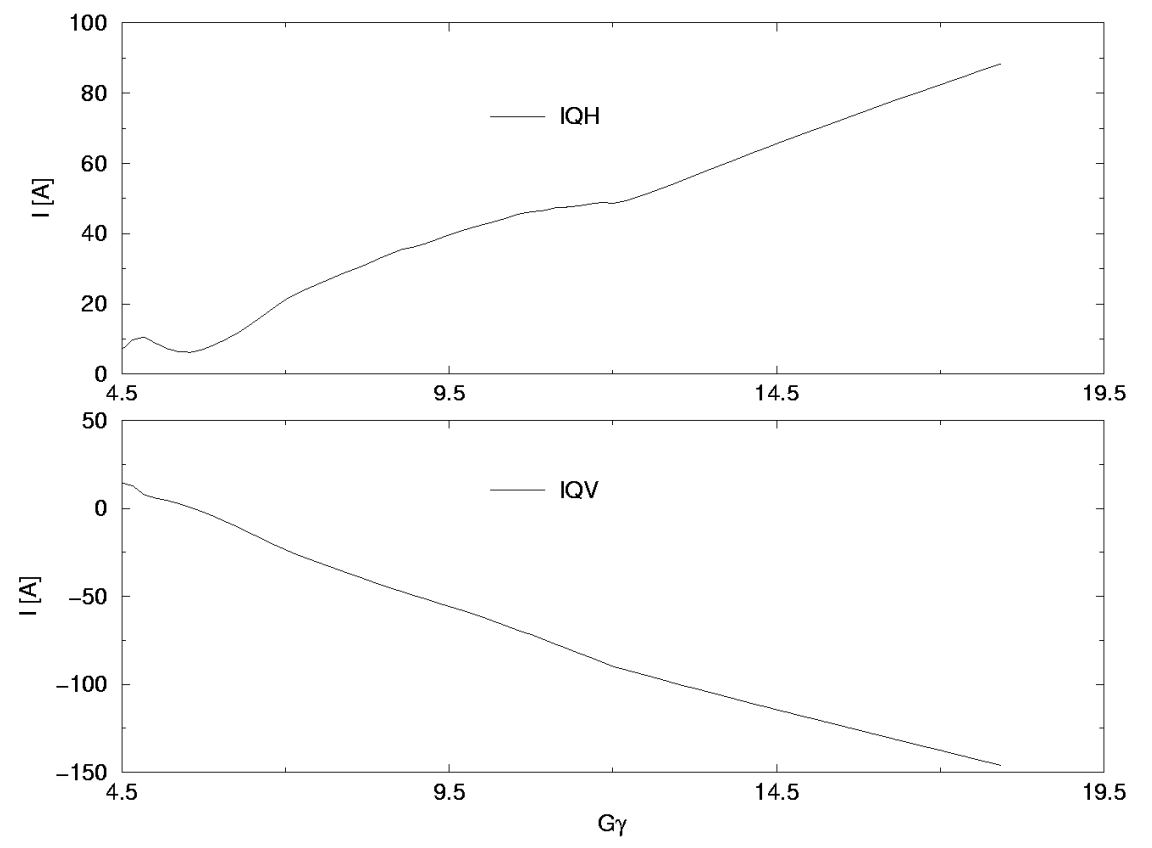

Figure 6. The current of the horizontal (top) and vertical (bottom) tune quadrupoles as function of $\mathrm{G} \gamma$. 


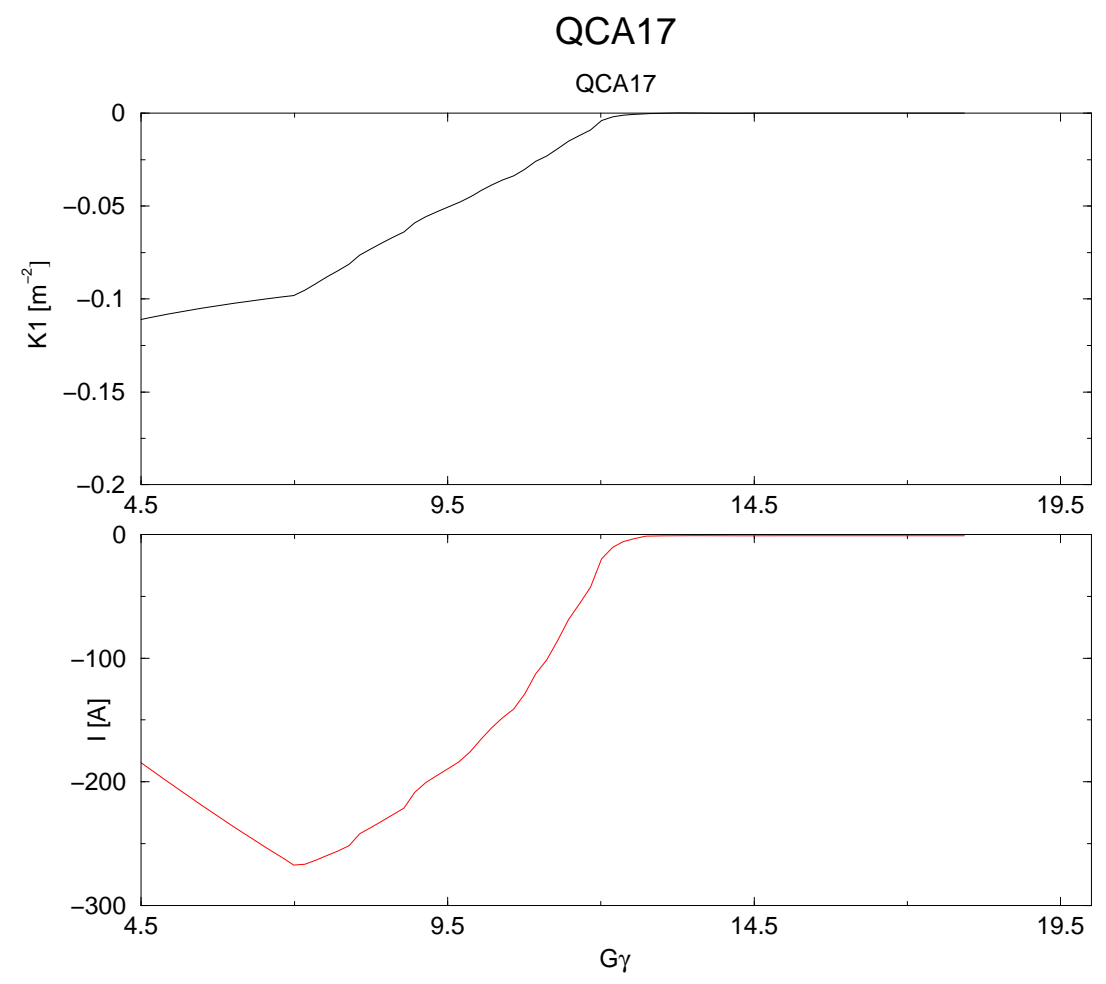

Figure 7. Plots of the K1 values (top) and the current (bottom) for the compansation quad CQA17. QCA19
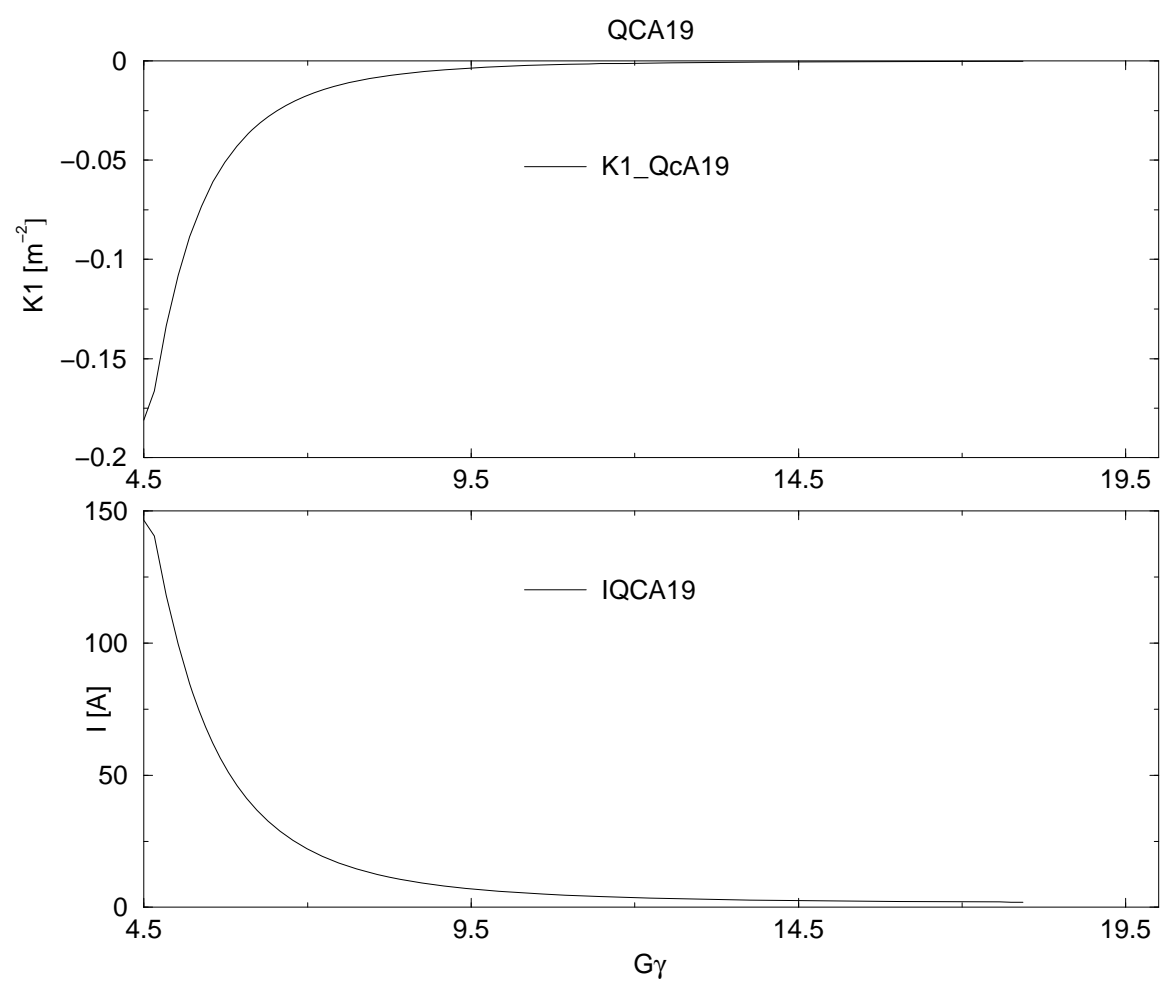
Figure 8. Plots of the K1 values (top) and the current (bottom) for the compensation quad CQA19.

QCB1

QCB1
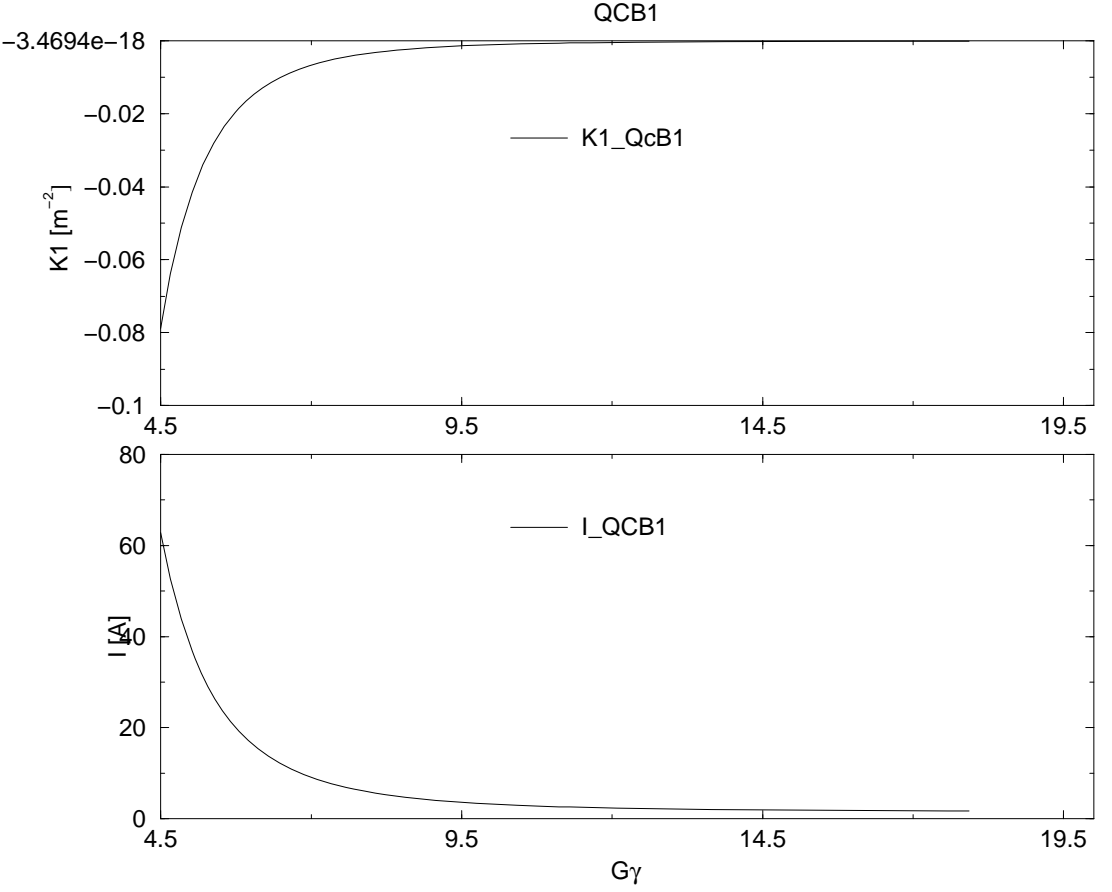

Figure 9. Plots of the K1 values (top) and the current (bottom) for the compensation quad CQB1.

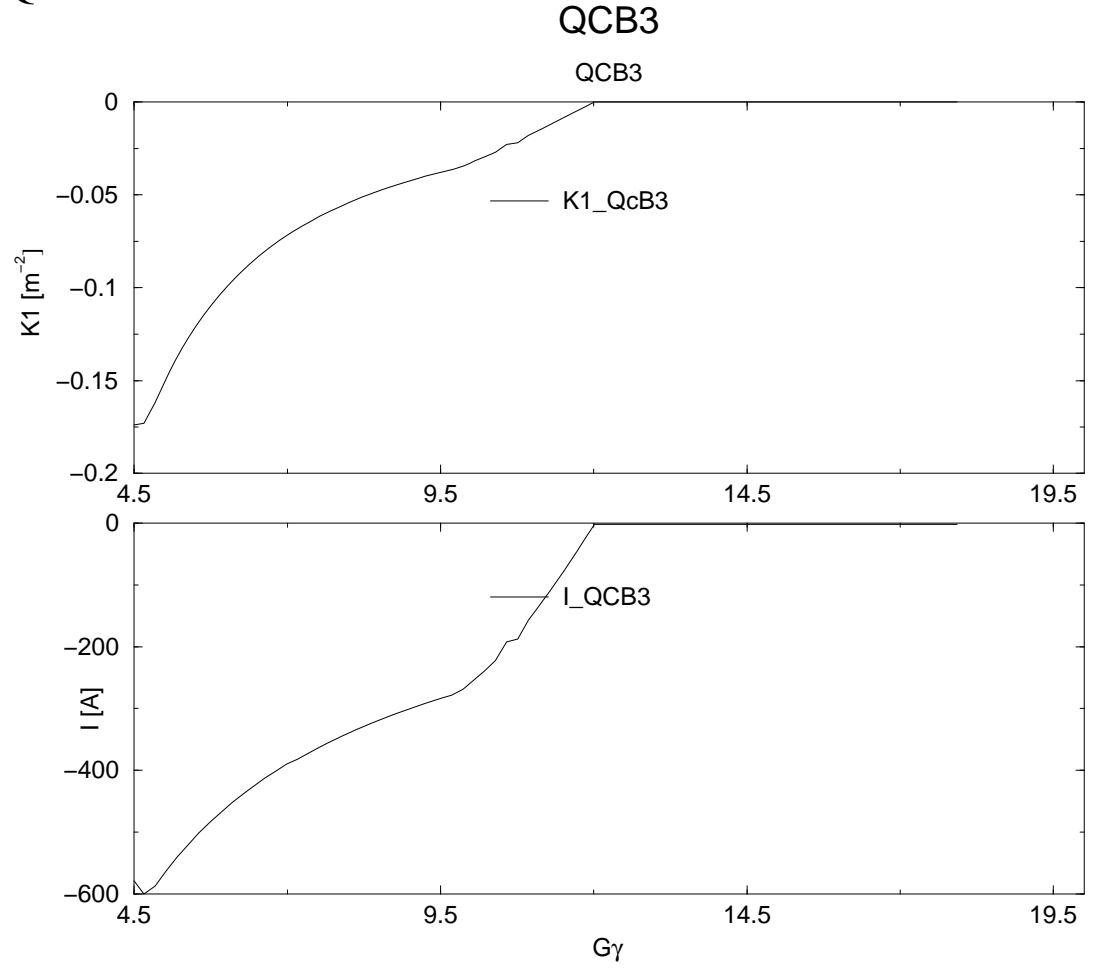


Figure 10. Plots of the K1 values (top) and the current (bottom) for the compensation quad CQB3. QCE17

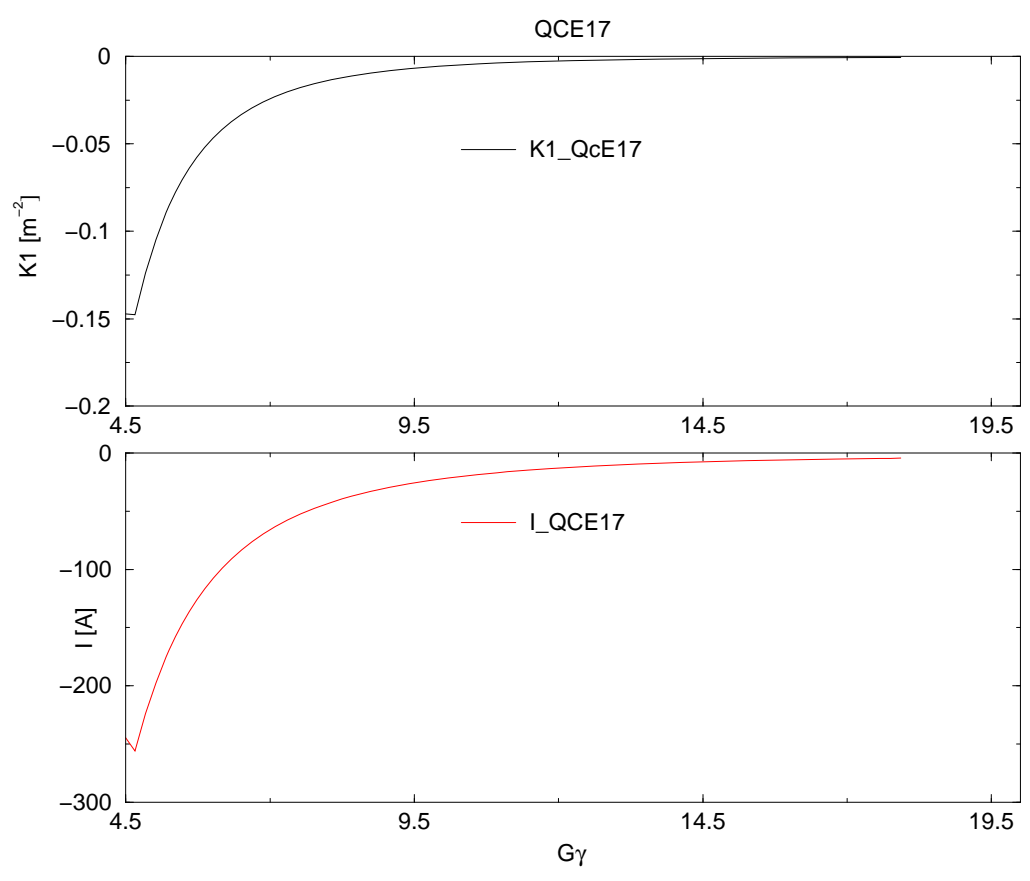

Figure 11. Plots of the K1 values (top) and the current (bottom) for the compensation quad CQE17.

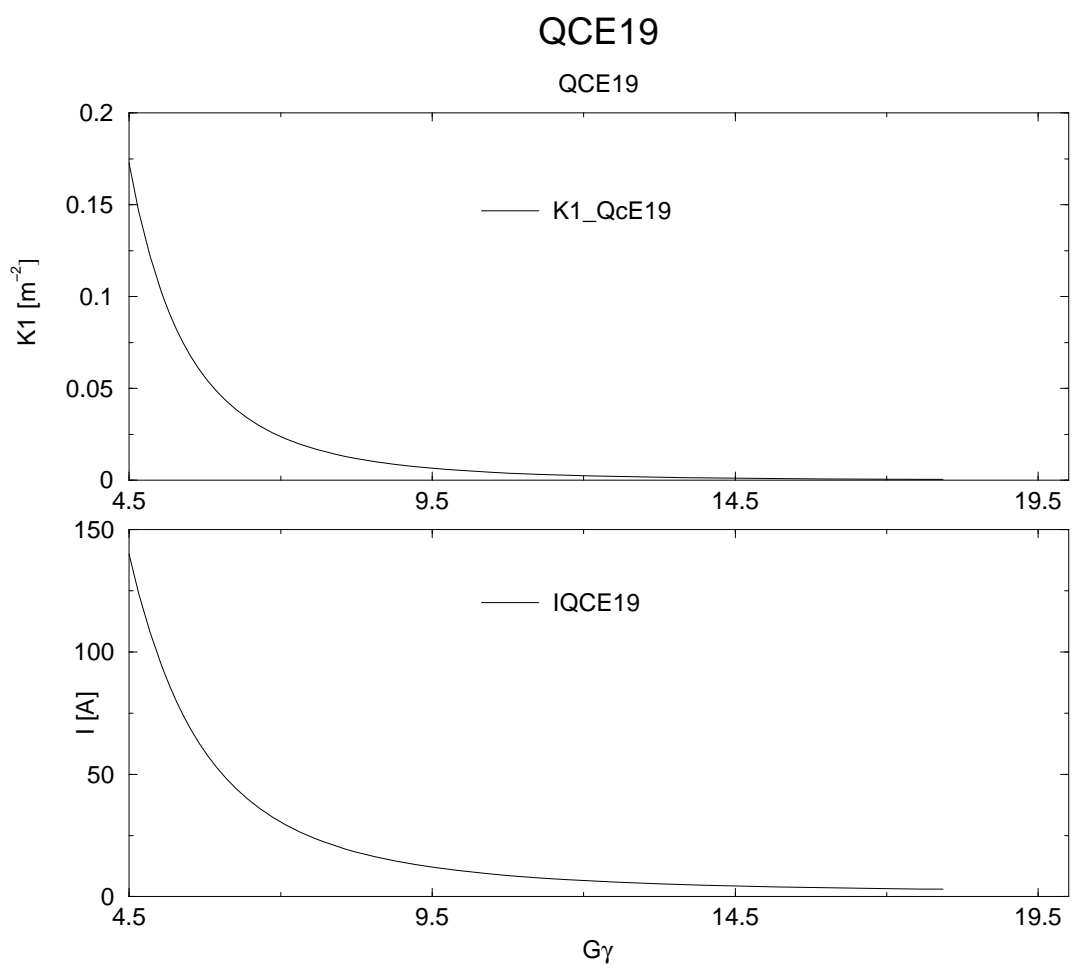


Figure 11. Plots of the K1 values (top) and the current (bottom) for the compensation quad CQE19. Since the compensation quad CQF1 is connected in series with the CQE19 the settings of the CQF1 are identical to those of CQE19, but with opposite polarity.

\section{Summary}

We report on the procedure we applied, to design the beam optics of the AGS during the acceleration of polarized protons. We also provide the calculated settings of the compensation quads and of the tune quads which satisfy the required beam constraints during the acceleration of polarized protons. Currently we are collecting experimental data in the AGS, when it runs with both helices, with the purpose to determine experimentally the optical properties of the helical magnets. It appears that the experimentally determined optics is not very different from the model calculated optics.

\section{References}

[1] T. Roser, et al., Proc. EPAC04, (2004), p. 1577

[2] H. Huang, et al., Proc. EPAC06, (2006), p. 273

[3] H. Huang, et al., PRL 99, 154801(2007)

[4] R. Gupta et al. "Magnet Design of a Superconducting Snake”, PAC03

[5] J. Takano et al. " Field Measurements in the AGS Warm Snake” EPAC04, 2116 (2004)

[6] E. Willen et al. ” Superconducting Helical Snake Magnet for AGS” PAC05, 2935 (2005)

[7] J. Takano et al. " Helical dipole partial siberian snake for AGS” S. Inst. P11002, (2006)

[8] N. Tsoupas et. al. Proc. PAC07, (2007) p. 3723

[9] V. Bargman, et. al. Phys. Rev. Lett. 2, 435 (1959)

[10] L.H. Thomas, Phil. Mag. 3,7, $\underline{3}$ (1927)

[11] Vector Fields Inc.

[12] The MAD Program CERN/LEP-TH/88-38 